\title{
Polarization-resolved conoscopic patterns of planar oriented liquid crystal cells
}

\author{
Roman G. Vovk, Alexei D. Kiselev \\ Institute of Physics of NASU, Prospect Nauki 46, 03028 Kiev, Ukraine
}

Received February 15, 2011; accepted March 14, 2011; published March 31, 2011

\begin{abstract}
We theoretically and experimentally investigate the angular structure of polarization of light transmitted through planar nematic and cholesteric liquid crystal (LC) cells by analyzing the polarization state as a function of the incidence angles and the polarization characteristics of the incident wave (the ellipticity $\varepsilon_{i n c}$ and the azimuth of polarization $\varphi_{\text {inc }}$. The geometry of polarization-resolved conoscopic (angular) patterns emerging after the planar nematic and cholesteric LC cells is described in terms of the polarization singularities such as C-points (points of circular polarization) and L-lines (lines of linear polarization). The characteristic feature of polarization-resolved conoscopic patterns under consideration is that the conditions of C-point formation are very sensitive to polarization parameters of the incident wave.
\end{abstract}

Liquid crystals are anisotropic materials. It is well known that the anisotropy of LCs is determined by their orientational structure, which is sensitive to external fields and boundary conditions [1]. The optical properties of LCs determine their numerous applications as materials with quite readily controllable anisotropy $[2,3]$ and play an important role in solving such problems as the characterization (i.e., determination of parameters) of the orientation structure (alignment) of LCs.

Conoscopy is widely used as an experimental technique to characterize orientational structures in nematic LC (NLC) cells [4]. But the use of crossed polarizers leads to a loss of information about the state of polarization of light transmitted through the LC cell. In principle, this information may be useful in developing new devices and techniques for improving the optical characterization of anisotropic materials.

In our recent papers $[5,6]$ we have shown that the polarization structure of conoscopic images can be represented by two-dimensional distributions of the Stokes parameters [7]. Such distributions, termed the polarization-resolved conoscopic patterns, were described in terms of polarization singularities: C-point (point of circular polarization) and L-lines (lines of linear polarization) [8]. In polarization distributions of random light fields, such singularities are known to be fundamentally important as stable topological defects [9].

The experimental and theoretical results obtained in $[5$, 6] give a complete description of conoscopic patterns in

\footnotetext{
*E-mail: roman.vovk@gmail.com
}

the case of homeotropically oriented nematic liquid crystals (NLCs) in which the orientation structure (with the NLC director aligned in the direction of normal to the substrate plane) is characterized by cylindrical symmetry with the axis of rotation perpendicular to the cell plane ( $z$ axis). In particular, we have studied in detail the bifurcation behavior of $C$-points during transformations of the polarization-resolved conoscopic patterns induced by a change in the polarization parameters (polarization azimuth and ellipticity) of the incident wave.

In studying the polarization distributions of light in other LC orientation structures, one may naturally expect the manifestation of effects that are directly related to breaking the cylindrical symmetry of the homeotropic configuration. In order to study these effects, we concentrate on a planar oriented LC layer as another important limiting case with the LC director n (determining the orientation of the optical axis) lies in the LC cell plane ( $x y$ plane) and is expressed as follows:

$$
\mathbf{n}=n_{x} \mathbf{x}+n_{y} \mathbf{y}=\cos \varphi_{d} \mathbf{x}+\sin \varphi_{d} \mathbf{y},
$$

where $\varphi_{d}$ is the azimuthal angle of the director. In addition to the polarization distributions of light in conoscopic patterns of a homogeneous NLC pattern with $\varphi_{d}=\varphi_{0}$, we also consider the case of cholesteric LCs with the helicoidal orientation structure described by Eq. (1) with $\varphi_{d}=q z+\varphi_{0}$, where $q=2 \pi / P$ and $P$ are the wavenumber and the pitch of the cholesteric helix, respectively [1]. In this letter, our task is to determine conditions of the formation of $C$-points in polarization distributions of this type.

Omitting mathematical details on deriving the exact solution to the problem of light transmission through an NLC cell, below we present the final analytical expression for the transmission matrix $\mathbf{T}$ (given by Eq. (2)) of a planar NLC cell in the general case of oblique incidence. In order to calculate the transmission matrix, we use the analytical results obtained in $[5,6]$, where the solution of the corresponding boundary-value problem was expressed via the operator of evolution of Maxwell's equations in the matrix form [10]. It should be noted that, for cholesteric LC (CLC) cells, similar analytical 
expressions can be written only in the case of normally incident light whereas, in the case of oblique incidence, the solution cannot be obtained in the closed form and the evolution operator has to be determined using numerical methods.

$$
\begin{gathered}
\left(\begin{array}{c}
E_{\|}^{(t r)} \\
E_{\perp}^{(t r)}
\end{array}\right)=\mathbf{T}\left(\begin{array}{c}
E_{\|}^{(i n c)} \\
E_{\perp}^{(i n c)}
\end{array}\right) \\
\mathbf{T}=N_{m}\left[\mathbf{A}_{+} \mathbf{W}_{-} \mathbf{A}_{+}^{\mathbf{T}}-\mathbf{A}_{-} \mathbf{W}_{+} \mathbf{A}_{-}^{\mathrm{T}}\right]^{-1}, \\
\mathbf{W}_{ \pm}=\exp [ \pm i \mathbf{Q} h] \mathbf{N}^{-1}, \mathbf{N}=\operatorname{diag}\left[N_{e}, N_{o}\right], \\
N_{e}=\frac{2 q_{e} \mu}{n_{o}^{2}}\left(n_{o}^{2}-q_{x}^{2} n_{x}^{2}\right), N_{o}=2 q_{o} \mu\left(n_{o}^{2}-q_{x}^{2} n_{x}^{2}\right), \\
\mathbf{A}_{ \pm}=\mathbf{E}_{\mathbf{m}} \mathbf{H} \pm \mathbf{H}_{\mathbf{m}} \mathbf{E},
\end{gathered}
$$

where $\quad \mathbf{Q}=\operatorname{diag}\left[q_{e}, q_{o}\right] ; \quad q_{e}=\sqrt{n_{e}^{2}-q_{x}^{2}\left(1+u_{a} n_{x}^{2}\right)}$, $q_{o}=\sqrt{n_{o}^{2}-q_{x}^{2}}, \quad q_{m}=\sqrt{n_{m}^{2}-q_{x}^{2}}, \quad q_{x}=n_{m} \sin \theta_{i n c}$; $N_{m}=2 q_{m} / \mu_{m} ; \quad h=k_{v a c} d, \quad k_{v a c}=\omega / c \quad$ is the wavenumber in vacuum and $d$ is the thickness of an LC cell; $u_{a}=\left(n_{e}-n_{o}\right) / n_{o}$ is the parameter of anisotropy of the NLC; $n_{e}, n_{o}, n_{m}$ is the refractive indexes of LC and surround medium; $\mu$ and $\mu_{m}$ is the magnetic permeability LC and medium; $\theta_{i n c}$ is the incidence angle.

$$
\begin{aligned}
& \mathbf{E}=\mu\left(\begin{array}{ll}
n_{x}\left[1-q_{x}^{2} / n_{o}^{2}\right] & n_{y} q_{o} \\
n_{y} & -n_{x} q_{o}
\end{array}\right), \\
& \mathbf{H}=\left(\begin{array}{ll}
n_{x} q_{e} & n_{y} n_{o}^{2} \\
n_{y} q_{e} & -n_{x}\left[n_{0}^{2}-q_{x}^{2}\right]
\end{array}\right)
\end{aligned}
$$

are block-matrices of the corresponding eigenvectors. Analogous matrices of eigenvectors for the external medium are written as follows:

$$
\mathbf{E}_{\mathbf{m}}=\operatorname{diag}\left(q_{m} / n_{m}, 1\right), \mu_{m} \mathbf{H}_{\mathbf{m}}=\operatorname{diag}\left(n_{m}, q_{m}\right) .
$$

Matrix $\mathbf{T}$ calculated in the plane of incidence with the azimuthal angle $\varphi_{i n c}=0$ determines the transmission matrix $\tilde{\mathbf{T}}$ for conoscopic patterns in the circular basis set as follows $[5,6]$ :

$\tilde{\mathbf{T}}(\rho, \varphi)=\exp \left(-i \varphi \boldsymbol{\sigma}_{3}\right) \mathbf{T}_{\mathbf{c}}\left(\rho, \varphi_{d}-\varphi\right) \exp \left(-i \varphi \boldsymbol{\sigma}_{3}\right)$, $\rho=r \tan \theta_{i n c}, \quad \varphi=\varphi_{i n c}$,

where $\boldsymbol{\sigma}_{3}=\operatorname{diag}(1,-1), \mathbf{T}_{\mathbf{C}}=\mathbf{C} \mathbf{T} \mathbf{C}^{*}, \mathbf{C}=2^{-1 / 2}\left(\begin{array}{ll}1 & -i \\ 1 & i\end{array}\right)$ and $r$ is the scaling factor. In the plane of observation of the conoscopic pattern, $\rho$ and $\varphi$ represent the polar coordinates which are determined by the angle of incidence $\theta_{i n c}$ and the azimuthal angle $\varphi_{i n c}$ of the plane of incidence.

The polarization-resolved conoscopic patterns can now be computed as distributions of polarization ellipses in the plane of observation. Parameters that define the polarization ellipse (polarization azimuth $\varphi_{p}$ and ellipticity $\varepsilon_{\text {ell }}$ ) [7] can be calculated by the following equations:

$$
\varphi_{p}=\frac{1}{2} \arctan \left[\frac{S_{2}}{S_{1}}\right], \varepsilon_{\text {ell }}=\tan \left[\arcsin \left(\frac{S_{3}}{S_{0}}\right)\right],
$$

where $\left\{S_{0}, S_{1}, S_{2}, S_{3}\right\}$ are the Stokes parameters [7] that determine the Poincare sphere parameterized by the polarization azimuth $\left(0<\varphi_{p}<\pi\right)$ and the ellipticity angle $\left(-1<\tan \chi_{p}=\varepsilon_{\text {ell }}<1\right)$.

Now let us consider the conditions for the formation of $C$-points in polarization-resolved conoscopic patterns. Since the transmission matrix $\tilde{\mathbf{T}}(\rho, \varphi)$ depends on the two angles of incidence $\left(\theta_{i n c}, \varphi_{i n c}\right)$, the $C$-point for the given values of these angles appears at certain polarization parameters of the incident wave. In order to determine these polarization states, which induce the formation of $C$-points, let us solve the inverse problem of light transmission. By varying the angles of incidence, we obtain a manifold of polarization states of the incident wave, which induces at least one $C$-point in the corresponding conoscopic pattern. These states are conveniently imaged as points determining a region on the Poincare sphere.
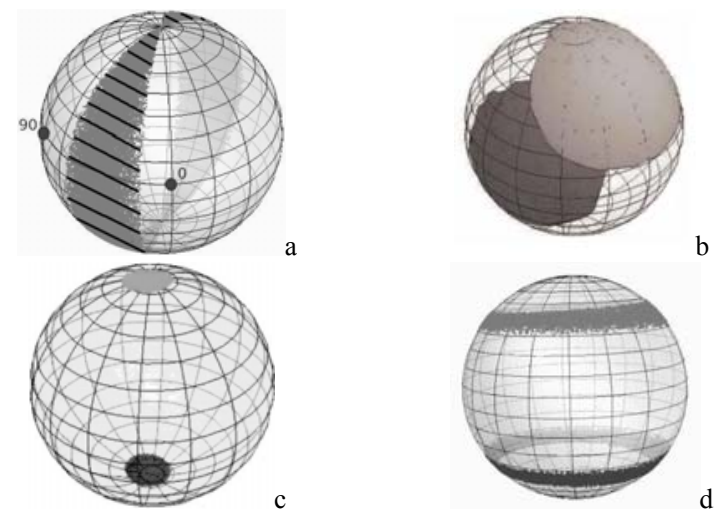

Fig.1. Polarization states of the incident wave, which induce $C$-points in the polarization-resolved conoscopic patterns, as mapped on the Poincare sphere for (a) planar NLC and (b-d) cholesteric LCs with helix pitches of $P=250,200$, and $100 \mu \mathrm{m}$, respectively. Bright and dark regions correspond to the polarization states inducing right- and left-handed $C$ points, respectively (for the planar NLC, these regions coincide). Parameters of calculations: LC, E7 $\left(n_{\mathrm{o}}=1.54 ; n_{\mathrm{e}}=1.72\right) ; n_{\mathrm{m}}=1.5 ;$ LC cell thickness, $d=100 \mu \mathrm{m}$; light beam aperture, $30^{\circ} ; \varphi_{\mathrm{d}}=0$. Direction of the polarization azimuth is indicated in Fig. 1a 
Figure 1a shows the regions of polarization states of the incident wave inducing $C$-points in the polarizationresolved conoscopic patterns for a planar NLC, as mapped on the Poincare sphere, where the polarization azimuth varies along the parallels and the ellipticity varies along the meridians. The parameters of calculations performed using Eq. (2)-(8) are given in the legend to Fig. 1. As can be seen from Fig. 1a, the LC with a planar orientation exhibits a pronounced dependence of the conditions of $C$-point formation on the azimuthal polarization angle of the incident wave. The Poincare sphere presented in this figure was calculated for $\varphi_{d}=0$.

A change in the azimuthal angle $\varphi_{d}$ of the NLC director is equivalent to a shift of the polarization azimuth by the same value of $\varphi_{d}$, which corresponds to translation of the region along the parallel (i.e., rotation around the $S_{3}$ axis).

Figure 2 presents the polarization-resolved conoscopic patterns for a crystal KDP which demonstrates selectivity with respect to the polarization azimuth of the incident wave. The $C$-points in these polarization-resolved conoscopic patterns form a regular geometric structure which is characterized by alternation of the sign of the topological index of $C$-points. The transformation of this structure under variations of the polarization azimuth is accompanied by bifurcations of the creation/annihilation of $C$-points and by the repulsion of structurally unstable intersections of $L$-lines.

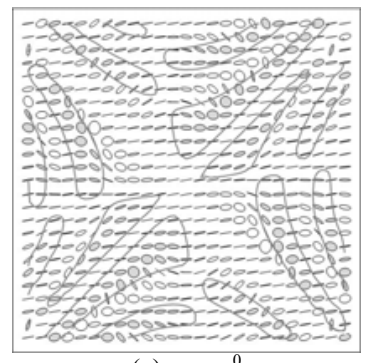

(a) $\varphi_{\text {inc }=20}{ }^{0}$

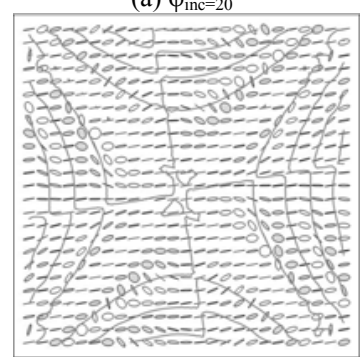

(c) $\varphi_{\text {inc }=20}{ }^{0}$

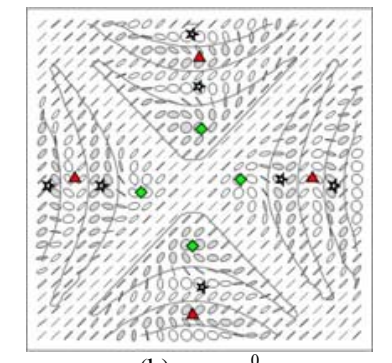

(b) $\varphi_{\text {inc }}=45^{0}$

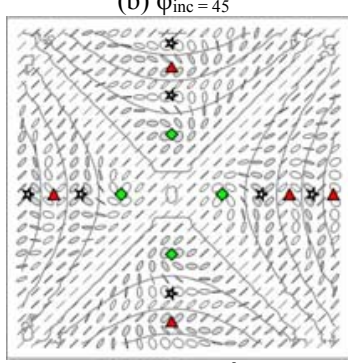

(d) $\varphi_{\text {inc }}=45^{0}$
Fig. 2. Experimentally measured (a, b) [6] and theoretically computed (c, d) the polarization-resolved conoscopic patterns. KDP, $d=10 \mathrm{~mm}$, $n_{o}=1.507, n_{e}=1.467$, light beam aperture $30^{\circ}$. L-lines are marked by solid lines. C-points are marked by a diamond (type Lemon), star (type Star) and triangle (type Monstar).
Figures $1 \mathrm{~b}-1 \mathrm{~d}$ show regions of the polarization states that induce $C$-points in the polarization-resolved conoscopic patterns of a CLC. Even in the first case (Fig. $1 \mathrm{~b})$, in which the helix pitch is $P=250 \mu \mathrm{m}$ and the number of half-turns is below one $(2 d / P=0.4)$, the regions representing polarization states that induce $C$-points of different handedness are separated. Similar to the above case of the NLC cell, cylindrical symmetry in this case is broken and the dependence on the azimuthal angle is retained. Here, it is possible to form $C$-points by varying the ellipticity of the incident light.

As it can be seen from Fig. 1c, a decrease in the CLC helix pitch to $P=200 \mu \mathrm{m}$ (with an increase in the number of half-turns to unity) leads to localization of the regions that induce $C$-points of opposite signs in the vicinity of the corresponding poles on the Poincare sphere. When the CLC cell accommodates two half-turns of the helix with $P=100 \mu \mathrm{m}$, these regions transform into two bands located in the upper and lower hemispheres (Fig. 1d).

It is interesting that, in the cases of CLC helices with integer numbers of half-turns considered above, the corresponding regions on the Poincare sphere become cylindrically symmetric so that the conditions of $C$-point formation no longer depend on the azimuthal angle. In these CLC cells, the appearance of $C$-points in the polarization-resolved conoscopic pattern is fully determined by the ellipticity of the incident light wave.

So, the characteristic feature of the polarization-resolved conoscopic patterns of planar nematic and cholesteric LC cells is selectivity of the conditions of $C$-point formation with respect to polarization parameters of the incident light wave. For planar NLCs, it is found that the appearance of $C$-points is determined entirely by the azimuthal polarization angle $\varphi_{p}^{(i n c)}$ whereas, for CLC cells, the control parameter is the ellipticity $\varepsilon_{\text {ell }}^{(i n c)}$.

\section{References}

[1] P.G. de Gennes, J. Prost, The Physics of Liquid Crystals (Clarendon, Oxford, 1993).

[2] P. Yeh, C. Gu, Optics of Liquid Crystal Displays (Wiley, Singapore, 1999).

[3] V.G. Chigrinov, Liquid Crystal Devices: Physics and Applications (Artech House, Boston, 1999).

[4] L.H. Brett, H.H. Winter, Appl. Opt. 40, 2089 (2001).

[5] A.D. Kiselev, J. Phys.: Condens. Matter 19, 246102 (2007).

[6] A.D. Kiselev, R.G. Vovk, R.I. Egorov, V.G. Chigrinov, Phys. Rev. A. 78, 033815 (2008).

[7] M. Born, E. Wolf, Principles of Optics (Pergamon, Oxford, 1980).

[8] J.F. Nye, Natural Focusing and Fine Structure of Light: Caustics and Wave Dislocations (Institute of Physics, Bristol, 1999).

[9] N.D. Mermin, Rev. Mod. Phys. 51, 591 (1979).

[10] D.W. Berreman, J. Opt. Soc. Am. 62, 502 (1972). 